\title{
Weakened Links Between Mind and Body in Older Age: The Case for Maturational Dualism in the Experience of Emotion
}

\section{Citation}

Mendes, Wendy Berry. Forthcoming. Weakened links between mind and body in older age: The case for maturational dualism in the experience of emotion. Emotion Review.

\section{Published Version}

doi:10.1177/1754073910364149

\section{Permanent link}

http://nrs.harvard.edu/urn-3:HUL.InstRepos:4214914

\section{Terms of Use}

This article was downloaded from Harvard University's DASH repository, and is made available under the terms and conditions applicable to Open Access Policy Articles, as set forth at http:// nrs.harvard.edu/urn-3:HUL.InstRepos:dash.current.terms-of-use\#OAP

\section{Share Your Story}

The Harvard community has made this article openly available. Please share how this access benefits you. Submit a story.

Accessibility 
Maturational dualism, 1

Weakened links between mind and body in older age:

The case for maturational dualism in the experience of emotion

\author{
Wendy Berry Mendes \\ Harvard University
}

In press: Emotion Review 


\begin{abstract}
Much of modern emotion research assumes reliable mind-body connectionschanges in emotional states influence bodily responses and, vice versa, somatovisceral information shapes emotional experiences. However, the ability to sense bodily states declines in older age as does activation of physiological systems, both of which may contribute to an impairment in mind-body connections. This essay is not intended to challenge the dominant view of embodiment, but rather provide evidence that weaker mind-body connections in older adults may have profound influences on emotional experiences and consequent behavior. I describe this phenomenon as maturational dualism and suggest implications of this for emotional experiences in older adults.
\end{abstract}


Weakened links between mind and body in older age:

The case for maturational dualism in the experience of emotion

There is little reason to be optimistic about how the aging process influences our brains and bodies. Cognitive declines such as deterioration in short term memory, reaction times, and attention occur even in the absence of neurological diseases (e.g., Levy, 1994). In the body, loss of muscle mass, deficiencies of growth hormones, hardening of the vasculature, and blunted activation reduces the flexibility of responding to different environmental demands (e.g., Epel, Burke, \& Wolkowitz, 2007; Matthews, 2005). Because bodily responses are often directly implicated in the experience of emotions, in this essay I explore some consequences of bodily changes brought on by aging in the experience of emotion.

Specifically, I review evidence of declines in sensory perception of the body with age and how this decline can interrupt the mind-body connection. I suggest that the weakened mind-body connection in older adulthood is primarily due to a loss of peripheral perception and blunted physiological reactivity. Due to these somatovisceral changes, the ability to use internal states to guide decisions and behavior may be impaired. Thus emotions, cognitions, and intentions may live in the mind (and brain) but not be embodied in the same way as they are in younger adults. I then describe the idea of maturational dualism, a phenomenon that suggests that the bodily changes that often cooccur with the aging process can influence the experience of emotion in specific ways. Maturational dualism challenges the idea that mind-body connections are stable across the life course and suggests that dissociations between mind and body have implications 
for emotions and consequent behavior and decision making, specifically, that older adults more than younger adults will rely on their external world to provide information regarding their emotional states. Some existing data are reviewed as evidence of this phenomenon and additional perspectives of this state are described.

The body's role in emotion

Though there are many disagreements in the emotion literature, a generally accepted principle is that emotions are embodied in some meaningful way. Embodiment theory suggests that a reciprocal relationship exists between peripheral bodily expressions and central nervous system processing of information from those domains (Niedenthal, Barsalou, Winkielman, Krauth-Gruber, \& Ric, 2005). To put it simply, the proverbial detached brain would be impoverished in terms of information processing without peripheral embodiments. Peripheral bodily expressions primarily take one of two forms in emotion research: 1) proprioception, the perception of the position of one's body in space, and 2) interoception, the perception of internal changes in the body. Both of these bodily expressions can influence emotional experience.

Proprioception. The idea that postural changes or manipulation of facial muscles related to expressions influences emotional experience is central to several empirical approaches in emotion research. For example, the directed facial action movement task requires participants to isolate muscles in the face and contract them so that a facial expression of emotion is configured and experiences of emotions concordant with the facial expression are then achieved (e.g., Ekman, 2007). Similarly, holding a pen with one's teeth activates the zygomaticus major muscle region (i.e., muscles associated with 
pulling back the lips to achieve a smile), which then facilitates happier ratings due to peripheral feedback loops (Strack, Martin, \& Stepper, 1988).

Moving away from the face, posture and body positioning can also influence emotional experience in animals and humans. In fear-conditioned rats, restraining the body during electric shock resulted in more threat/withdrawal physiology (i.e., moderate increases in cardiac responses and increased blood pressure) whereas unrestrained rats showed more approach physiology during shocks (i.e., increased cardiac activation and no changes in blood pressure) (Iwata \& LeDoux, 1988). Even though the "emotion" was the same (fear) the body positioning influenced the physiological responses that would facilitate freezing versus escaping.

In a recent paper, reminiscent of the Iwata \& LeDoux study, Harmon-Jones \& Peterson (2009) highlighted the importance of body positioning in the experience of emotion. To illustrate, imagine you are lying in bed next to your spouse and you start to argue. You may have the impulse to sit upright when you are arguing. Sitting upright would facilitate action and is thus more concordant with the motivational orientation of anger (Carver \& Harmon-Jones, 2009). If the postural position was inconsistent with anger (e.g., lying down) this might dampen the emotional response. To test the importance of congruent postural positioning for the experience of emotion, participants either sat upright or reclined while they heard an insult (or neutral feedback) from another "student" while electroencephalogram activity was recorded. Consistent with embodiment theory, and specifically proprioceptive influences, when participants were upright and "angry" they showed shifts in greater left frontal cortical activation compared to the upright-neutral condition. Importantly, the comparison of recline-insult to upright- 
insult was also significantly different. When participants were reclined and insulted they did not show shifts in left frontal cortical activation. Thus, postural changes blunted the emotional reaction.

Interoception. The ability to sense visceral organ activity, interoception, is implicated in the experience of emotion as well. For example, Hantas, Katkin, and Blascovich (1982) found that more accurate heart beat detectors self-reported greater emotional reactions to emotionally evocative photographs than inaccurate ones. More recently, Barrett, Quigley, Bliss-Moreau and Aronson (2004) proposed that visceral interoceptive ability was positively related to the intensity of emotional experience as delineated in Barrett's core affect model (Barrett \& Bliss-Moreau, 2009). They observed that participants with weaker interoception were less likely to make distinctions between emotions of different arousal intensity.

It seems that proprioception and interoception are important for the experience of emotion, but is there evidence that these sensory modalities decline with age like other senses such as taste, hearing and vision? The short answer is, yes, both proprioception and interoception decline with old age. In a recent paper, Khalsa and colleagues (2009) used a heart beat detection paradigm with participants ranging in age from 22 to 63 . Older subjects, on average, showed poorer detection of their heart beats than younger and middle aged adults, and the overall bivariate correlation between age and accurate heart beat detection was $r=-.49$ and $r=-.45$ at two time points. These effects persisted after controlling for BMI, sex, and the ability to detect one's own pulse directly from the wrist. With regard to proprioceptive declines with age, a much larger literature exists documenting these effects. Indeed a recent review article (Goble, et al., 2009) provides 
evidence of proprioceptive impairments with age along varied dimensions of static and dynamic body positions.

Blunted physiological reactivity. For the past two decades Levenson and colleagues have examined physiological reactivity during emotional experiences among older adults relative to younger adults (e.g., Levenson, et al., 1991). Across a number of studies they report a blunting of sympathetic nervous system responses in older adults. For example, they find lower heart rate responses for anger, fear, and sadness in older adults compared to younger adults during a directed facial action task. In some cases younger adults had twice as large SNS increases as older adults (a finding that mirrors physical exercise). Indeed, this observed blunting of SNS activity led Levenson and colleagues to suggest that "emotional experience may become more cognitive over time."

Importantly, in one of the Levenson paper's older adults did not show increases in peripheral (finger) skin temperature during anger evocations (Levenson, et al., 1991). Changes in the flexibility of the vasculature especially in the peripheral regions-arms and hands; legs and feet—are typically affected by neuropathy that can co-occur with age and the extremities tend to be affected first. The lack of skin temperature increases during anger is especially interesting given that anger is characterized as having an approach orientation (Carver \& Harmon-Jones, 2009) and has been associated with greater dilation of the arterioles allowing more blood to get to the effector muscles and periphery, which is one of the likely physiological changes that increases skin temperature during anger (Mendes, et al., 2008). However, it is important to note that the flexibility of the vasculature is compromised in an asymmetrical form with age - vessels can still constrict easily but are harder to dilate. Therefore, emotions that are more approach-oriented, like 
anger, may be compromised before emotions that are more threat/withdrawal related, such as fear. In sum, changes in the body that are impaired with age include interoception, proprioception, and blunted sympathetic nervous system, all of which are implicated in emotion embodiment.

\section{Maturation dualism}

Maturational dualism is the idea that the weakening of mind-body connections with age can influence emotional experiences. The aging process reduces interoceptive sensitivity primarily through increased somatization, which influences sensitivity and blunts perception of activation and deactivation in the emotional experience. Without perception of arousal as a component of the emotional experience, valence might be all that is left (see Barrett, et al., 2004). If interoception declines with age then the ability to differentiate emotions - especially emotions that differ in arousal—might be especially impaired resulting in fewer distinctions of emotional experience.

What are the possible emotional and behavioral consequences of loss of mindbody connections in older age? There are intriguing clues in the literature regarding how the disconnection in older age may influence behavioral responses. For example, one study examined the somatic marker hypothesis in older adults (Denburg, Tranel, \& Bechara, 2004). In previous papers, Damasio and his colleagues (e.g., Bechara, Damasio, Tranel, \& Damasio, 1997) described the somatic marker hypothesis, which suggested that bodily states outside of conscious awareness could influence behavior. To support this hypothesis participants (brain damaged and control) were presented with four decks of cards with various gains and losses associated with the cards. Two of the decks resulted in overall losses - large gains, but large losses as well, whereas the other two decks 
resulted in smaller gains, but smaller losses. They found that as participants turned over cards from the various decks, changes in skin conductance (activity in the eccrine gland, indicating sympathetic activation innervated by acetylcholine) co-occurred with choices from the riskier decks. Importantly, these bodily changes preceded conscious reporting of which decks were risky by approximately 40 trials. Thus, the somatic marker hypothesis claims that bodily changes can indicate psychological or mental states prior to conscious reporting. In this original article normal participants were compared to patients with ventral medial lesions. While normal participants consciously reported which decks were risky by about the $40^{\text {th }}$ trial, lesion patients were not able to learn this pattern.

In the extension of this earlier study with older adults (defined as 56 to 85 years old) similar to patients with VM lesions, older participants, on average, did not show preferences for the advantageous decks across five trials (or 100 cards) (Denburg, et al., 2004). When examining individual responses the authors reported that among the younger group 37 out of 40 participants eventually picked from the advantaged deck, among the older group only 15 out of 40 showed this same "unimpaired" pattern. The remaining older participants either showed more preference for the disadvantaged deck or no preference. There are at least two possible interpretations of these data in light of the ideas presented here: 1) similar to Levenson's data older participants had blunted physiological responses during the task, which limited the ability to sense internal states vis-á-vis the somatic marker hypothesis, or 2) the sympathetic response was intact and as strong as that experienced by younger participants, but the ability to sense the bodily changes - interoceptive awareness — was diminished (Khalsa, 2009). Of course another 
possibility is the lack of choice of the advantageous decks was due to a combination of blunted reactivity and loss of interoception.

It might be intuitive to assume that these deficits in older age are due to changes in brain structure, but a recent article cautions against a purely brain-based explanation of interoception (Khalsa, Rudrauf, Feinstein, \& Tranel, 2009). Khalsa and colleagues suggest that interoceptive awareness may not be tied exclusively to the insula and ACC as suggested by others (e.g., Craig, 2009). In this study a single patient with bilateral insula and ACC damage was given dosage level increases of isoproterenol-a sympathetic agonist similar to epinephrine - and was asked to self-report on his heart rate changes. Similar to control participants the patient both showed dosage level increases in heart rate and reported awareness of this change. Following this the experimenters then applied a topical anesthetic on the chest skin covering the heart rate sensation region. With the skin area anesthetized the patient could no longer report increases in heart rate, whereas control subjects could still report dosage level increases. This finding underscores the importance of considering multiple pathways to interoception, and especially bodily sensations.

An implication of maturational dualism is that individuals would have to rely more on the external environment to determine their internal states. This is consistent with Cartensen's socio-emotional selectivity theory, which describes a positivity effect in older adults including a shifting away from negative stimuli toward more positive stimuli and favoring positive and avoiding negative emotions (Cartensen, 2006).

Another implication of maturational dualism is that older participants would be more susceptible to suggestions of an emotional state since they might have to rely more 
on their external world to provide information about their internal states. Although recent theory and evidence suggest that environmental cues can strongly influence emotional states and meaning (Barrett, 2009; Jamieson, et al., 2009) the loss of exquisite ability to detect internal states should make older participants more susceptible to environmental cues. Imagine the Harmon-Jones study described earlier with supine body position dampening the EEG activation. It would be expected that to the extent that the dampening of the approach response was due to sensing one's body in spaceproprioception - the expectation is that the supine versus upright position would have less of an impact on the emotional experience in older adults.

\section{Theoretical and practical considerations}

This essay was intended to draw from existing data and theory to examine how mind-body relations differ across the life span and how that might influence the experience, perception, and consequences of emotions. Though I have focused on describing the phenomenon and some evidence supportive of the idea, this perspective potentially has practical purposes as well. Although one can imagine that there might be some advantage to becoming less sensitive to internal states as we age to protect us from debilitating pain, there are obviously some serious downsides of not having strong mindbody connections, especially when considering how the connection benefits emotional experience. Most critically, to the extent that the bodily responses that co-occur with emotions tend to provide signals on how to behave (e.g., approach, avoid, or freeze) the lack of association between the mind and body might impair the ability to make good decisions. 


\section{References}

Barrett, L. F. (2006). Solving the emotion paradox: Categorization and the experience of emotion. Personality and Social Psychology Review, 10, 20-46.

Barrett, L. F., \& Bliss-Moreau, E. (2009). Affect as a psychological primitive. Advances in Experimental Social Psychology, 41, 167-218.

Barrett, L. F., Quigley, K. S., Bliss-Moreau, E., \& Aronson, K. R. (2004). Interoceptive sensitivity and self-reports of emotional experience. Journal of Personality and Social Psychology, 87, 684-697.

Damasio, H., \& Tranel, D. (1997). Deciding advantageously before knowing the advantageous strategy, Science, 275, 1293-1294.

Carstensen, L. L. (2006). The influence of a sense of time on human development. Science, 312, 1913-1915.

Carver, C.S. \& Harmon-Jones, E. (2009). Anger is an approach-related affect: Evidence and implications. Psychological Bulletin, 135, 183-204.

Craig, A.D. (2009). How do you feel - now? The anterior insula and human awareness. Nature Reviews Neuroscience, 10, 59-70.

Denburg, N. L., Tranel, D., \& Bechara, A. (2005). The ability to decide advantageously declines prematurely in some normal older persons. Neuropsychologia, 43, 10991106.

Ekman, P. (2007). The directed facial action task: Emotional responses without appraisal. In: J. A. Coan, \& J. J. B. Allen (Eds.), Handbook of emotion elicitation and Assessment (pp. 47-53). New York, NY: Oxford University Press.

Epel, E., Burke, H., \& Wolkowitz, O.M. (2007). The psychoneuroendocrinology of 
aging: anabolic and catabolic hormones. In: C. M. Aldwin, C.L. Park, \& A. Spiro (Eds.), Handbook of health psychology and aging (pp. 119-141). New York, NY: Guilford Press.

Goble, D. J., Coxon, J. P., Wenderoth, N., Van Impe, A., \& Swinnen, S. P. (2009). Proprioceptive sensibility in the elderly: Degeneration, functional consequences and plastic-adaptive processes. Neuroscience and Biobehavioral Reviews, 33, 271-278.

Hantas, M., Katkin, E.S., \& Blascovich, J. (1982). Relationship between heart-beat discrimination and subjective experience of affective state. Psychophysiology, 19, 563.

Harmon-Jones, E., \& Peterson, C. K. (2009). Supine body position reduces neural response to anger evocation. Psychological Science, 20, 1209-1210.

Iwata, J. \& LeDoux, J. E. (1988). Dissociation of associative and nonassociative concomitants of classical fear conditioning in the freely behaving rat. Behavioral Neuroscience, 102, 66-76.

Jamieson, J., Mendes, W. B., Blackstock, E., \& Schmader, T. (2009). Turning the knots in your stomach into bows: Reappraising arousal improves performance on the GRE. Journal of Experimental Social Psychology, 46, 208-212.

Khalsa, S. S., Rudrauf, D., Feinstein, J.S., \& Tranel, D. (2009). The pathways of interoceptive awareness. Nature Neuroscience, 12, 1494-1496.

Khalsa, S. S., Rudrauf, D., \& Tranel, D. (2009). Interoceptive awareness declines with age. Psychophysiology, 46, 1130-1136.

Levenson, R. W., Carstensen, L. L., Friesen, W. V., \& Ekman, P. (1991). Emotion, 
physiology, and expression in old age. Psychology and Aging, 6, 28-35.

Levy, R. (1994). Aging-Associated Cognitive Decline. In International Psychogeriatrics, (pp.63-68). Cambridge University Press.

Matthews, K. A. (2005). Psychological perspectives on the development of coronary heart disease. American Psychologist, 60, 783-796.

Mendes, W. B., Major, B., McCoy, S., \& Blascovich, J. (2008). How attributional ambiguity shapes physiological and emotional responses to social rejection and acceptance. Journal of Personality and Social Psychology, 94, 278-291.

Niedenthal, P.M., Barsalou, L.W., Winkielman, P., Krauth-Gruber, S., \& Ric, F. (2005). Embodiment in attitudes, social perception, and emotion. Personality \& Social Psychology Review, 9, 184-211.

Strack, F., Martin, L. L., \& Stepper, S. (1988). Inhibiting and facilitating conditions of the human smile: A nonobtrusive test of the facial feedback hypothesis. Journal of Personality and Social Psychology, 54, 768-777. 